INGENIERÍA QUÍMICA

\title{
Cinética de flotación de la materia orgánica en carbones del Cerrejón (Guajira) utilizando una columna de flotación
}

\section{Kinetics of flotation of organic matter in Cerrejón coals (Guajira) using a flotation column}

\author{
J Piñeres*, J Barraza*, M Solarte*, M Orozco, P Colon** $§$ \\ *Escuela de Ingeniería Química, Universidad del Valle, Cali, Colombia \\ **Carbones del Cerrejón Limited, Bogotá, Colombia \\ §luispin@univalle.edu.co,juan.barraza@correounivalle.edu.co \\ angeliksolarte@gmail.com,machela26@hotmail.com \\ pedro.Colon@cerrejoncoal.com
}

(Recibido: Noviembre 09 de 2011 - Aceptado: Noviembre 19 de 2012)

\begin{abstract}
Resumen
Este trabajo muestra las constantes cinéticas de tres carbones bituminosos del Cerrejón (Guajira), M1, M2 y M3. Se utilizó una columna de flotación a escala de laboratorio ( $5 \mathrm{~m}$ de altura y $5 \mathrm{~cm}$ de diámetro) a condiciones normales de $\mathrm{pH}$ (7.2), usando como reactivos de flotación: Lauril Eter Sulfato de Sodio al 28\% (p/p) como espumante con una concentración de $15.4 \mathrm{lb} / \mathrm{t}$ de carbón y aceite de creosota como colector con una concentración de $10 \mathrm{lb} / \mathrm{t}$ de carbón, empleando diferentes tiempos de recolección de muestras (se tomaron 5 muestras de flotados cada $45 \mathrm{~s}$ para un total de $3.75 \mathrm{~min}$ ). Los resultados mostraron una cinética de primer orden para la recuperación de material combustible. Las constantes cinéticas calculadas se encontraron en el rango de 0.126 a $0.255 \mathrm{~min}^{-1}$, presentando los mayores valores la muestra M3. La recuperación de material combustible presentó un rango entre 42 y $61 \%$, la muestra de carbón que mostró mayor recuperación de material combustible fue la muestra M3. En general, se obtuvieron reducciones de cenizas entre 71 y $91 \%$, lo que indica que las condiciones de operación utilizadas en el proceso y la liberación de ceniza fueron favorables.
\end{abstract}

Palabras clave: Columna de flotación, Constante cinética, Recuperación de material combustible

\begin{abstract}
This work shows the kinetic constants of three bituminous coals of Cerrejón (Guajira), M1, M2 and M3. A flotation column at laboratory scale $(5 \mathrm{~m}$ high and $5 \mathrm{~cm}$ in diameter) at normal $\mathrm{pH}(7.2)$ was used as flotation reagents, Sodium Lauryl Ether Sulfate $28 \%(\mathrm{w} / \mathrm{w})$ as a frother with a concentration of $15.4 \mathrm{lb} / \mathrm{t}$ of coal and creosote oil as a collector with a concentration of $10 \mathrm{lb} / \mathrm{t}$ of coal was used, using different sample collection times (five floated samples were taken every $45 \mathrm{~s}$, for a total of $3.75 \mathrm{~min}$ ). The results showed first-order kinetics constant for the recovery of combustible material. The results showed first-order kinetics constant for combustible recovery material. The kinetic constants calculated are in the range of $0126-0255 \mathrm{~min}^{-1}$, M3 sample showed the highest values. The combustible recovery material are in the range of $42-61 \%$, M3 sample coal showed greater recovery of combustible material. In general, ash reductions were obtained between 71 and $91 \%$, indicating that the operating conditions used in the process and the ash release was favorable.
\end{abstract}

Keywords: Combustible recovery, Flotation column, Kinetic constant. 


\section{Introducción}

Un desarrollo significativo en el proceso de flotación con el transcurrir de los años es el incremento en el uso de las columnas de flotación en diversas industrias. Una columna de flotación se diferencia dramáticamente de una celda convencional en su diseño y filosofía de operación, siendo atractiva en aquellos procesos de limpieza donde se requieren múltiples etapas, debido a que una columna de flotación puede remplazar múltiples celdas de flotación, generando un circuito más simple y fácil de controlar, Piñeres \& Barraza (2008).

Actualmente, las columnas de flotación son ampliamente utilizadas en los procesos de limpieza de carbón, basándose principalmente en las diferencias de las propiedades superficiales de sus componentes. Durante la flotación, las partículas con superficie hidrofóbica se adhieren a las burbujas de aire (materia orgánica) y son llevadas a la corriente de flotados (en el tope de la columna), en tanto que las partículas hidrofílicas (materia mineral) se obtienen en la corriente de descarga (en el fondo de la columna) Piñeres \& Barraza (2008).

El carbón es un material extremadamente heterogéneo compuesto de materiales orgánicos e inorgánicos. La fracción orgánica la representa los macerales, en tanto que a la fracción inorgánica la conforman la materia mineral y azufre. La flotabilidad de la fracción orgánica depende de varios factores tales como la composición petrográfica, grado de carbonización, oxidación de la superficie, entre otros (Leonard, 1991; Blaschke, 1996).

La evaluación de la constante cinética de flotación es un parámetro necesario para el cálculo de la longitud de una columna de flotación, así como para la determinación de sus condiciones de mezclado. En general, los principios de la cinética química son normalmente usados en el modelado del proceso de flotación, particularmente, la ecuación básica de velocidad representada por la siguiente expresión, (Dobby \& Finch, 1986; Finch \& Dobby, 1990):

$$
\frac{d C}{d t}=-k C^{n}
$$

donde $C$, en el caso de una reacción química, representa la concentración del reactivo, $t$ es el tiempo, $k$ es la constante de velocidad y $n$ es el orden de la reacción. En el caso de la flotación de partículas hidrofóbicas, puede utilizarse una ecuación similar a la que se usa en reacciones químicas, cambiando $C$ por $m$ que es la masa de las partículas flotables remanentes en la celda.

La similitud entre las ecuaciones para $C$ y $m$ se justifica por la suposición de que el volumen dentro de la columna de flotación permanece constante. Para columnas de flotación que operan con bajos flujos de aire y pequeños diámetros de burbuja el modelo de dispersión en flujo pistón hace una buena descripción del mezclado dentro de la columna. Sin embargo, el número de dispersión se calcula asumiendo que el coeficiente de dispersión axial de las partículas es equivalente al del líquido. Para condiciones de flujo pistón y cinética de primer orden, (Dobby \& Finch, 1986; Finch \& Dobby, 1990), presentan la siguiente ecuación:

$$
R=1-\exp \left(-k \tau_{p}\right)
$$

donde $\tau_{\mathrm{p}}$ representa el tiempo de residencia del sólido, $k$ es la constante de velocidad y $\mathrm{R}$ es la recuperación fraccional del material flotado.

El objetivo de este trabajo fue evaluar experimentalmente las constantes cinéticas de flotación de tres muestras de carbón (M1, M2 y M3) procedentes de la mina el Cerrejón, usando una columna de flotación a escala piloto, con la finalidad de diseñar y construir columnas de flotación para limpieza de carbones colombianos.

\section{Metodología}

\subsection{Materiales}

Tres muestras (M1, M2 y M3) de carbones Colombianos procedentes de la mina el Cerrejón fueron utilizadas. Las muestras (tal como fueron recibidas) se sometieron a un proceso de molienda hasta obtener malla $200(75 \mu \mathrm{m})$, como resultado de un estudio de liberación, (Orozco \& Solarte, 2010). Aceite de creosota, lauril éter sulfato de sodio al $28 \%$, se utilizaron como colector y espumante respectivamente. 


\subsection{Equipo}

La columna de flotación utilizada, tiene $5 \mathrm{~m}$ de altura y un diámetro de $0.05 \mathrm{~m}$, construida en acrílico. La entrada del flujo de alimentación se ubicó a $1.2 \mathrm{~m}$ medidos desde el tope de la columna, el sistema de generación de burbuja, que consta de un burbujeador poroso fabricado en vidrio, se colocó a $0.38 \mathrm{~m}$ medidos desde el fondo de la columna. Así mismo, la entrada del flujo de aire se ubicó a la misma altura, en tanto que, la entrada del flujo de espumante se ubicó a $0.66 \mathrm{~m}$ del fondo de la columna. La recolección del material flotado se realizó por medio de un vertedero ubicado a $0.22 \mathrm{~m}$ del tope, el cual tiene un diámetro de $0.25 \mathrm{~m}$, una altura de $0.40 \mathrm{~m}$ de frente, y $0.20 \mathrm{~m}$ por detrás de la columna. En la Figura 1 se muestra un esquema del equipo utilizado.

\subsection{Procedimiento experimental}

Inicialmente se agregó agua en el tanque de alimentación y se adicionó el colector a la concentración deseada, $10 \mathrm{lb} / \mathrm{t}$ de carbón, posteriormente se adicionó la cantidad específica de carbón, lo cual produce una pulpa con concentración del $2.5 \% \mathrm{p} / \mathrm{p}$ de carbón en todas las pruebas. Esta pulpa se mezcló durante 30 min hasta obtener una mezcla homogénea, luego se abre la válvula de alimentación hasta obtener el flujo deseado. Cuando la mezcla alcance $0.23 \mathrm{~m}$ aproximadamente por encima de la alimentación se abre la válvula del rotámetro de aire asegurándose que el manómetro indique 14.7 psi y el flujo de aire sea el deseado. Luego se ajustan los flujos de espumante y agua, para mantener la altura deseada por encima de la alimentación. Las corrientes de tope y fondo se recogen en recipientes y posteriormente se filtran.

Las muestras de carbón se secaron y almacenaron para realizarles los análisis necesarios, durante las corridas se tomaron 5 muestras de flotados cada $45 \mathrm{~s}$ para un total de $3.75 \mathrm{~min}$. Todas las corridas se realizaron usando una sola etapa.

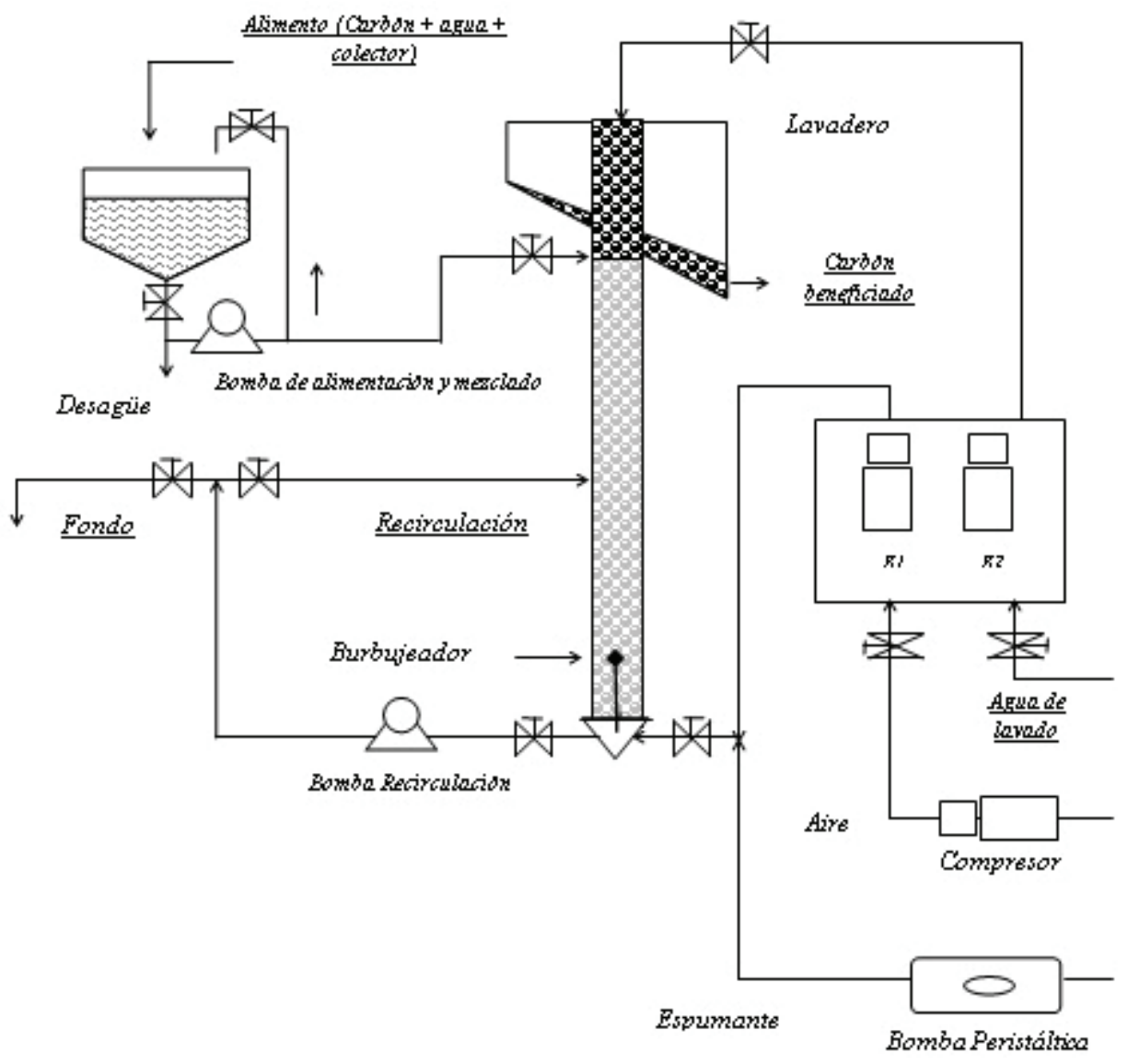

Figura 1 Diagrama de la columna de flotación a escala piloto. R1:Rótametro de aire, R2:Rótametro de agua 
Para incrementar el tiempo de permanencia de las partículas, un flujo de recirculación se colocó a $0.65 \mathrm{~m}$ medidos desde el punto de alimentación. Se trabajó con un flujo de aire de $35.1 \mathrm{ml} / \mathrm{s}$, en tanto el flujo de alimentación se mantuvo constante en $26.28 \mathrm{ml} / \mathrm{s}$ y el flujo de fondo se ajustó a $27.18 \mathrm{ml} / \mathrm{s}$, garantizando la altura deseada de $0.23 \mathrm{~m}$ sobre la alimentación de la pulpa, en tanto el flujo de espumante se mantuvo constante en $42.19 \mathrm{ml} / \mathrm{min}$, con una concentración de $15.4 \mathrm{lb} / \mathrm{t}$ de carbón. Todas las corridas se realizaron a pH normal de la pulpa el cual fue de 7.2, con un flujo de agua de lavado de $2.2 \mathrm{ml} / \mathrm{s}$. El error experimental está basado en experimentos repetidos y fue de $\pm 5 \%$.

\section{Resultados y discusión}

Caracterización de las muestras: En la Tabla 1 se muestran los resultados de los análisis próximos de las muestras M1, M2 y M3, realizados acorde con las normas ASTM. Con los resultados encontrados y con la clasificación del rango de carbones ASTM, se encontró que los carbones del Cerrejón son de tipo bituminoso alto en volátiles (Leonard, 1991). Como se observa en la Tabla 1, la muestra M3 presenta el mayor contenido de cenizas $(25.44 \%)$, lo cual se refleja en el menor contenido de carbón fijo (42.28\%) y menor poder calorífico (10160 $\mathrm{Btu} / \mathrm{lb}$ ). Adicionalmente esta muestra presenta el mayor contenido de azufre total. Por el contrario la muestra M1, presenta el menor contenido de cenizas $(4.2 \%)$, lo cual también se refleja en su mayor poder calorífico (13484 Btu/ 1b). La muestra M1 también presenta el menor contenido de azufre. En términos del contenido de cenizas, poder calorífico y azufre, la muestra M2 presentó un comportamiento intermedio entre las muestras M1 y M3.

Tabla 1. Análisis próximo de las muestras alimentadas a la columna de flotación, - 200M base seca (bs).

\begin{tabular}{cccc}
\hline & M1 & M2 & M3 \\
\hline Materia volátil, \% & 39.74 & 38.43 & 32.29 \\
Cenizas, bs\% & 4.20 & 7.80 & 25.44 \\
Carbón Fijo, \% & 56.06 & 53.77 & 42.28 \\
Poder Calorífico, Btu/lb & 13484 & 13104 & 10160 \\
Azufre total, bs\% & 0.54 & 0.55 & 0.83 \\
\hline
\end{tabular}

Determinación de la constante cinética: En la determinación de la constante cinética de flotación, se utilizó la Ec. (2) bajo condiciones de flujo pistón en la columna. Sobre la base de los resultados de rendimiento másico, recuperación orgánica y porcentaje de cenizas en función del tiempo de flotación se aplicó la Ec. (2) para obtener valores de $-\ln (1-\mathrm{R})$ vs $\tau_{\mathrm{p}}$ de flotación. Estos valores se procesaron y se presentan en la Figura 2 para las tres muestras M1, M2 y M3.

Como se observa de la Figura 2, para las tres muestras de carbón (M1, M2 y M3) se obtuvo un coeficiente de correlación $\left(\mathrm{r}^{2}\right)$ mayor a 0.92 , indicando que la cinética de flotación es de primer orden. Estos resultados están de acuerdo con otros estudios similares reportados en la literatura, Piñeres \& Barraza (2008) y Piñeres et al., (2009).

Acorde a los valores de las pendientes obtenidas en las tres líneas presentadas en la Figura 2, se observa que la mayor constante cinética $(0.255$ $\mathrm{min}^{-1}$ ) de recuperación de materia orgánica en los flotados se encontró para la muestra M3. Para las muestras M1 y M2 se obtuvieron valores de 0.139 y $0.126 \mathrm{~min}^{-1}$ respectivamente. Esto indica que la muestra M3 presentó la mayor tasa de flotación, en este estudio cinético (a pesar de presentar el mayor contenido de ceniza del alimento). En las Figuras 3 - 5 se graficaron los porcentajes de rendimiento másico, recuperación de materia orgánica y cenizas en función del tiempo de flotación para las muestras M1, M2 y M3.

En general, para las tres muestras se observa que el rendimiento másico de flotación y recuperación de material orgánico aumentan con el tiempo de flotación. Valores superiores al $45 \%$ de recuperación orgánica (sobre la base de la materia orgánica del alimento) fueron obtenidos en las tres muestras. Para el caso de las muestras M1 y M2 se observó que los rendimientos másicos y la recuperación orgánica son similares para todos los tiempos de flotación, sin embargo, la muestra M3 presentó diferencias entre los dos índices, siendo para todos los tiempos, las recuperaciones orgánicas mayores que los rendimientos másicos. Estas diferencias 


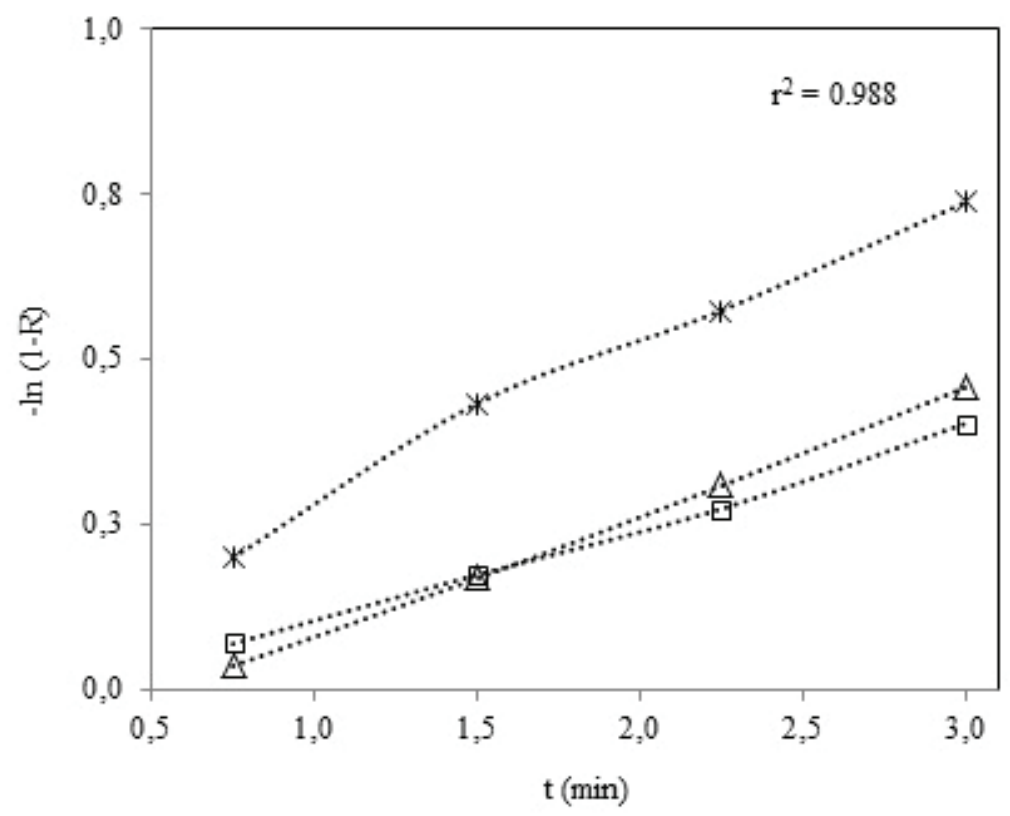

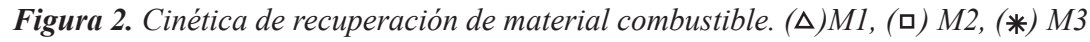

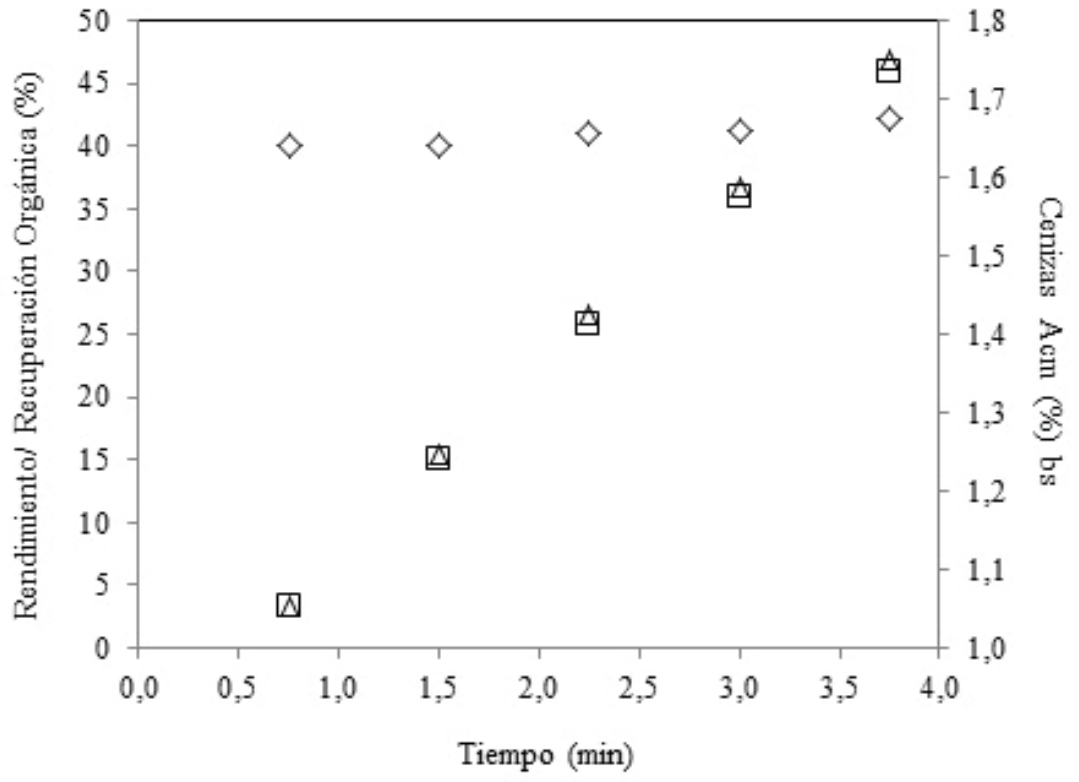

Figura 3. Porcentaje de recuperación orgánica, rendimiento y cenizas. $(\square)$ Rendimiento, $(\Delta)$ Recuperación Orgánica, ( $)$ Cenizas.

se deben a que la muestra M3 presenta un alto contenido de cenizas en comparación con las muestras M1 y M2.

Analizando el comportamiento del porcentaje de cenizas (base seca, bs) se observa que las muestras flotadas M1 y M2 presentaron valores alrededor de $1.7 \%$ para todos los tiempos de flotación, mientras que la muestra M3 presentó valores alrededor de $3.0 \%$. Esto significa que reducciones de ceniza de más del $71 \%$ fueron obtenidos en las muestras M1 y M2, mientras que para la muestra M3 presentó una reducción de cenizas del $91 \%$.

Las diferencias en los valores del porcentaje de recuperación de materia orgánica y reducción cenizas se deben principalmente a las 


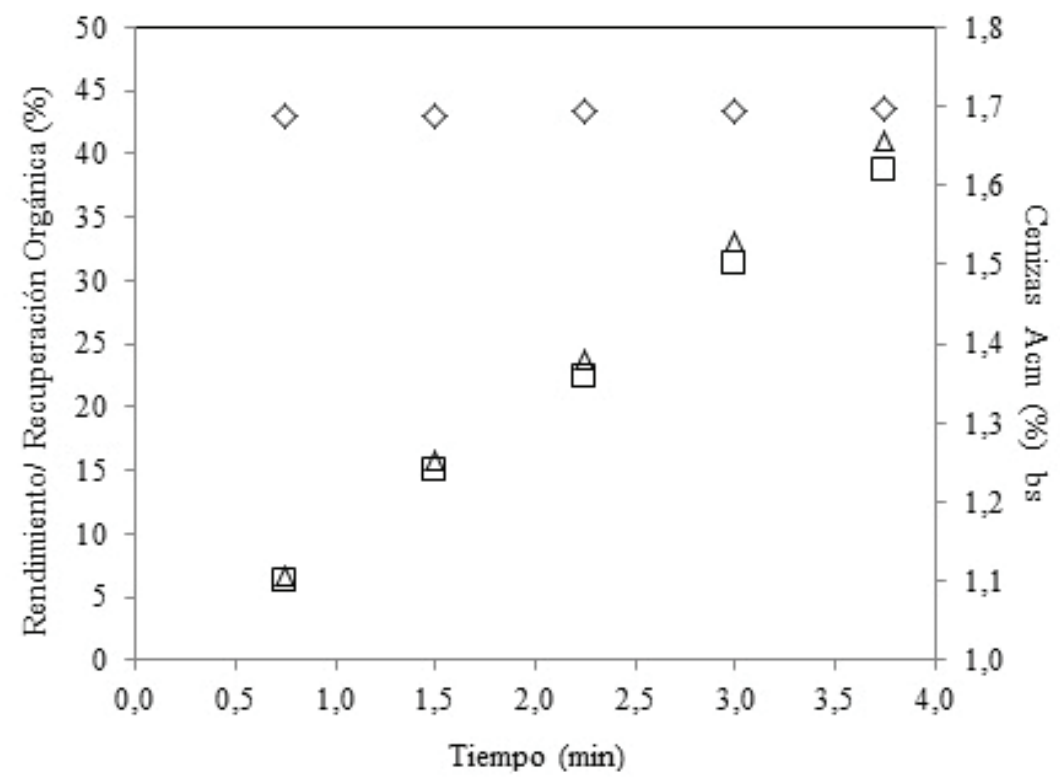

Figura 4. Porcentaje de recuperación orgánica, rendimiento y cenizas. (ם)Rendimiento, ( $\Delta$ ) Recuperación Orgánica, ( ) Cenizas.

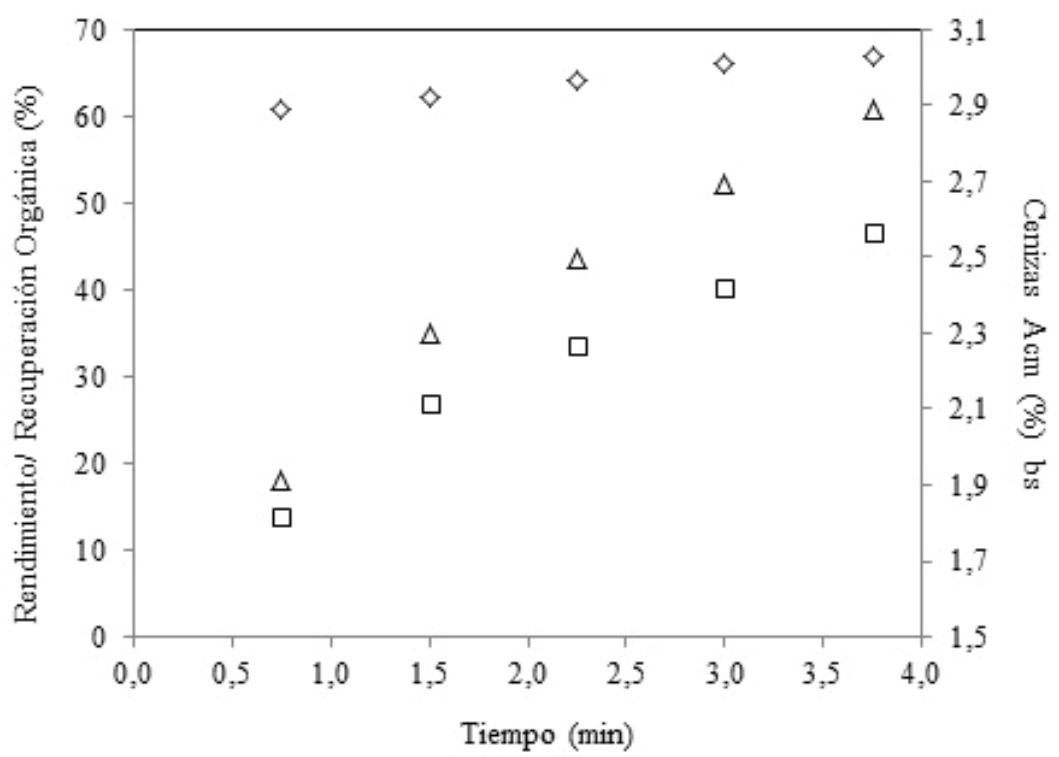

Figura 5. Porcentaje de recuperación orgánica, rendimiento y cenizas. (ם)Rendimiento, ( $\Delta$ ) Recuperación Orgánica, ( $)$ Cenizas.

diferencias en la flotabilidad de cada muestra de carbón como consecuencia de los distintos grados de carbonización, (Tabla 1) composición petrográfica, grupos funcionales, distribución mineralógica, modo y tipo de ocurrencia de estos minerales. Otros factores tales como las propiedades superficiales de las muestras de carbón, hidrodinámica de la columna de flotación, oxidación, grupos funcionales que contienen oxígeno de cada muestra de carbón, así como también las asociaciones entre los macerales y la materia mineral afectan su flotabilidad y selectividad, Piñeres (2008).

\section{Conclusiones}

En general, las muestras M1, M2 y M3 presentaron una cinética de flotación de 
material orgánico de primer orden. La muestra M3 presentó el mayor valor de las constantes cinéticas para flotar materia orgánica.

En general, para las tres muestras se obtuvo un elevado porcentaje de reducción de cenizas, teniendo en la muestra M3 el mayor valor, 91\% y las muestras M1 y M2 con un porcentaje de reducción de 71 y $79 \%$, respectivamente, indicando que la columna de flotación utilizada es selectiva para separar partículas finas hidrofóbicas (materia orgánica) de las hidrofílicas (materia mineral).

Las diferencias en los valores de las constantes cinéticas se deben principalmente a las diferencias en la flotabilidad de cada carbón como consecuencia de los distintos grados de carbonización, composición petrográfica, grupos funcionales, distribución mineralógica, modo y tipo de ocurrencia de estos minerales.

\section{Agradecimientos}

Los autores ofrecen sus agradecimientos al Instituto Colombiano de Ciencia y Tecnología (Colciencias) y a Carbones del Cerrejón Limited por el apoyo económico a través del proyecto "Licuefacción directa de carbones enriquecidos en el maceral vitrinita procedentes de la mina El Cerrejón (Guajira-Colombia)".

\section{Referencias bibliográficas}

Blaschke, W. S. (1996). New trends in coal preparation technologies and equipment. Kraków, Polonia. Gordon and Breach Publishers.
Dobby, G. S., \& Finch, J. A. (1986). Flotation column scale-up and modelling. Canadian Institute of Mining, Metallurgy and Petroleum (CIM) Bulletin 79 (889), 89-96.

Finch, J.A., \& Dobby, G. S. (1990) Column flotation. Toronto Canadá. Pergamon Press

Leonard, J. W. (1991). Coal preparation. Littleton Colorado, USA. Society for Mining, Metallurgy, and Exploration, Inc.

Orozco, M., \& Solarte, M. (2010). Cinética de flotación del maceral vitrinita en carbones del cerrejón (guajira) utilizando columna de flotación. Tesis Pregrado, Escuela de Ingeniería Química, Universidad del Valle, Cali, Colombia.

Piñeres, J., \& Barraza, J. (2008). Efecto del pH, velocidad del aire y concentración de espumante sobre la cinética de flotación de cuatro carbones colombianos. Ingeniería y competitividad 10 (2), 29-40.

Piñeres, J., Barraza, J., \& Blandón. (2009). Constantes cinéticas de flotación del grupo maceral vitrinita de dos carbones colombianos, Revista Ingeniería e Investigación 29 (3), 29 35.

Piñeres, J. (2008). Fenómenos superficiales $y$ cinéticos en la concentración del grupo maceral vitrinita en fracciones beneficiadas de carbones colombianos obtenidas por flotación burbujeante. Tesis de Doctorado, Escuela de Ingeniería Química, Universidad del Valle, Cali, Colombia. 